\title{
Granulomatöse Fremdkörperreaktion nach Augmentation von Aknenarben - eine diagnostische und therapeutische Herkulesaufgabe
}

\author{
Granulomatous Foreign Body Granuloma as a Reaction to Previous Augmentation of Acne Scars - \\ A Diagnostic and Therapeutic Hercules Challenge
}

Autoren

Institut
S. Psaier, C. Pföhler, T. Vogt, C. S. L. Müller

Klinik für Dermatologie, Venerologie und Allergologie, Universitätsklinikum des Saarlandes

\section{Bibliografie}

DOI http://dx.doi.org/

10.1055/s-0030-1256578

Akt Dermatol 2011; 37:

258-260 @ Georg Thieme

Verlag KG Stuttgart · New York

ISSN 0340-2541

Korrespondenzadresse

Dr. med. Cornelia S. L. Müller

Oberärztin der Dermatopathologie

Universitätsklinikum

des Saarlandes

Klinik für Dermatologie,

Venerologie und Allergologie

Kirrberger Str. 1

66421 Homburg/Saar

cornelia.mueller@uks.eu

\section{Zusammenfassung \\ $\checkmark$}

Für die Augmentationsbehandlung der Haut stehen diverse Materialien zur Verfügung. Ursächlich für auftretende unerwünschte Fremdkörperreaktionen sind in den meisten Fällen die genuinen Materialeigenschaften der Fillersubstanz und/oder eine falsche Injektionstechnik. Die therapeutischen Optionen sind bei einmal manifesten Granulomen enttäuschend. Bei inflammatorischen Granulomen sind eine topische und systemische Antibiose sowie topische Immunmodula-

\section{Einleitung}

Es wird über eine 45-jährige Patientin berichtet, welche sich mit seit ca. 6 Monaten bestehenden, rötlichen, gruppierten Knötchen an der linken Wange vorstellte. Bei zunächst negativer Anamnese konnte erst histologisch die Diagnose einer granulomatösen Reaktion auf eingebrachtes Fremdmaterial gestellt werden. Da die Injektionen des Dermafillers über 10 Jahre zurücklagen, war von Seiten der Patientin zunächst kein $\mathrm{Zu}$ sammenhang mit den aktuellen Hautveränderungen in Betracht gezogen worden. Die Therapie der Filler-Granulome stellte sich auch insofern als schwierig heraus, dass zum einen nach über 10 Jahren keine Unterlagen zum Eingriff und damit der Art des verwendeten Fillers mehr dokumentiert waren, zum anderen, da noch keine einheitlichen Richtlinien zur Therapie der Fillergranulome statuiert wurden.

\section{Anamnese}

$\nabla$

Eine 45-jährige Patientin stellte sich mit seit ca. 6 Monaten bestehenden, rötlichen, gruppierten Knötchen an der linken Wange sowie einem kleineren Knötchen an der rechten Wange vor toren angezeigt, bei fluktuierenden Läsionen Inzision und Drainage der betroffenen Areale. Vielversprechend scheinen Therapieansätze mit Lasersystemen (Diodenlaser für granulomatöse Läsionen sowie Lithium-Triborat (LBO)-532-nmLaser für inflammatorische Reaktionen) zu sein. Wir stellen eine asiatische Patientin vor, bei welcher vor weit über zehn Jahren eine Augmentationsbehandlung von Aknenarben an beiden Wangen durchgeführt wurde, und werden die wesentlichen diagnostischen Schritte sowie therapeutischen Optionen erläutern.

( $\bullet$ Abb. 1). Bislang waren erfolglos Lokaltherapien mit Ammoniumbituminosulfonat-Lösung und Prednicarbat-Creme sowie systemische Antibiosen mit Minocyclin und Doxycyclin durchgeführt worden. Keine internistischen Vorerkrankungen, keine Einnahme von Dauermedikamenten, keine bekannten Allergien, keine Auslandsaufenthalte in den vorausgehenden Monaten. Aknetherapien hätten nach Angaben der Patientin in der Vergangenheit nicht stattgefunden.

\section{Dermatologischer Befund}

An der Wange links zeigten sich mehrere gruppiert angeordnete, rötlich-livide, erhabene, prallelastische Knötchen. Ein weiteres, flacheres Knötchen zeigte sich auch an der rechten Wange ( $\bullet$ Abb. 1). Nebenbefundlich konnten multiple Aknenarben im Gesicht gesehen werden. Das restliche Integument zeigt sich unauffällig.

\section{Dermatopathologische Befunde}

Das histologische Bild zeigt in der HE-Färbung unterhalb einer unauffälligen Epidermis in der gesamten Dermis und Subkutis ausgeprägte kno- 


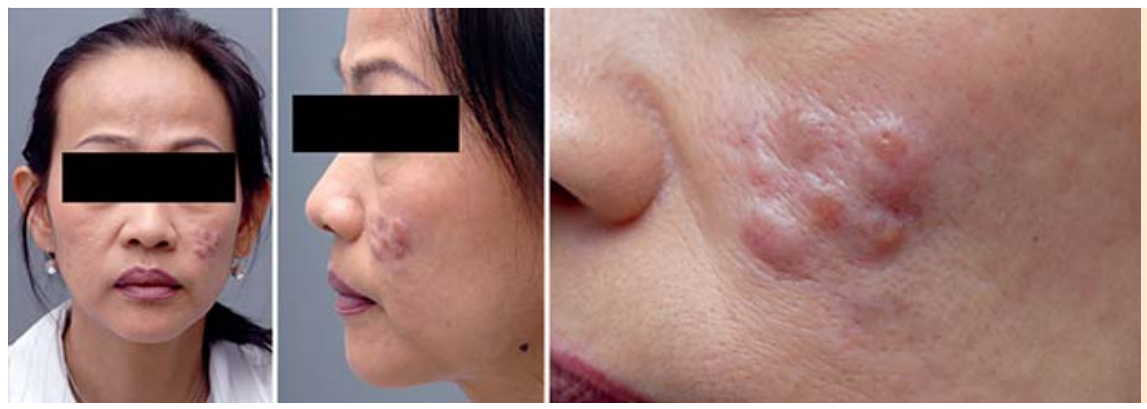

Abb. 1 Klinischer Befund bei Erstvorstellung der Patientin, welcher sich innerhalb von 6 Monaten manifestierte: gruppiert stehende, indolente, rötlich-livide Noduli an der Wange links.

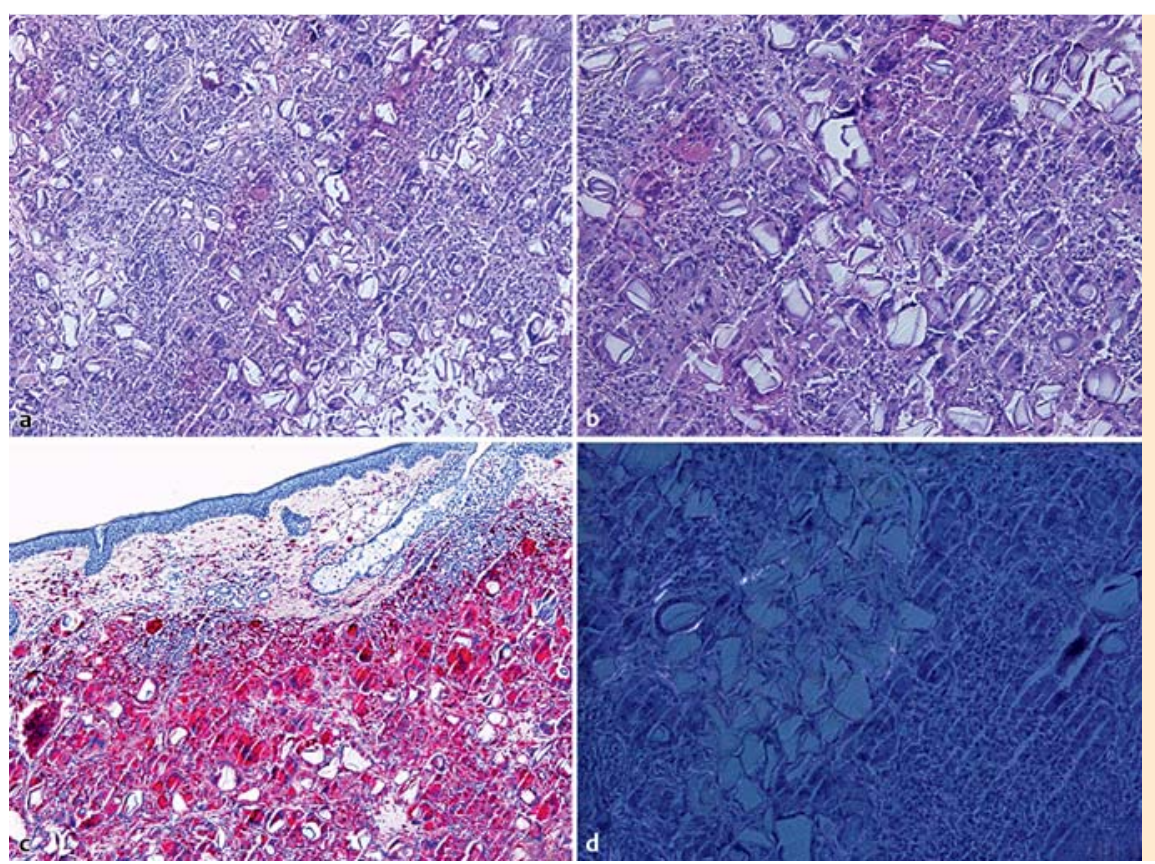

Abb. 2 Dermatohistologische Befunde in einer 4 -mm-Stanzbiopsie von der linken Wange: ausgedehnte, knotige, granulomatös-histiozytäre Infiltrate in Dermis und Subkutis, welche polarisationsoptisch nicht doppelbrechende kuboidale Fremdkörper umschließen. a, b Hematoxylin-Eosin; c Darstellung der Histiozyten im Infiltrat mittels CD68-Färbung; d polarisationsoptische Darstellung.

tige, granulomatös-histiozytäre Entzündungsinfiltrate mit zahllosen mehrkernigen Riesenzellen, welche kuboidale, transluzierende, polygonale Fremdkörper umschließen ( $\bullet$ Abb. 2a, b, c). Polarisationsoptisch keine Doppelbrechung des Fremdmaterials (๑ Abb.2d).

\section{Therapie und Verlauf \\ $\nabla$}

Erste therapeutische Versuche orientierten sich zunächst auf antiphlogistische/antigranulomatöse Lokaltherapien in Form von Methylprednisolonaceponat (Advantan ${ }^{\circledR}$ )-Milch $2 \times \operatorname{tgl}$. für die Dauer von 4 Wochen und Tacrolimus (Protopic ${ }^{\circledR}$ ) $0,03 \% 2 \times \operatorname{tgl}$. über 20 Wochen. Eine Abblassung der Rötung und Abwandlung in eine subakut-entzündliche, bräunlich-livide Veränderung konnte hierunter erzielt werden, der knotige Aspekt wurde jedoch nicht verbessert. In der Folge kombinierten wir die Lokaltherapie mit Allopurinol $300 \mathrm{mg}$ täglich per os. Bei weiterer unzureichender Besserung wurde diese Therapie nach 9 Wochen beendet. Es folgten intraläsionale Injektionen mit Triamcinolonacetonid $(10 \mathrm{mg} / \mathrm{ml}), 1: 1$ gemischt mit Mepivacain in zunächst wöchentlichen, später in zwei- und dreiwöchigen Abständen in Kombination mit Clarithromycin $500 \mathrm{mg} 2 \times \operatorname{tgl}$. per os über 6 Wochen. Es resultierten eine Abflachung der Knötchen und ein weiterer Rückgang des Erythems. Hingegen manifestierten sich zunehmend Komedonen der zugrunde liegenden Akne im Bereich der behandelten Areale, weshalb schlussendlich eine anti- komedogene Therapie mit Isotretinoin $10 \mathrm{mg}$ täglich mit den intraläsionalen Steroidinjektionen kombiniert wurde. Der weitere Verlauf bleibt abzuwarten.

\section{Diskussion}

Die Überweisung der Patientin an unsere Ambulanz erfolgte unter dem Verdacht auf eine Sarkoidose oder ein Granuloma eosinophilicum faciei. Die abschließende Diagnose konnte nur durch die eindrückliche Histologie gestellt werden. Ergänzend erfolgte eine insistierende Anamneseerhebung, bei der die Patientin schließlich angab, sich vor über zehn Jahren an einer Pariser Klinik für ästhetische Medizin einer Augmentationsbehandlung ihrer atrophen Aknenarben unterzogen zu haben. Der seinerzeit verwendete Dermafiller konnte bis dato nicht eruiert werden.

Für die Augmentationsbehandlung der Haut (Falten, Volumenverlust, Gewebedefekte und Narbenzüge) stehen heute diverse Materialien zur Verfügung. Die Einteilung der verschiedenen Fillersubstanzen erfolgt nach Materialien, Ursprung der Materialien, Bestandteilen, Handling, Effektdauer, Wirkmechanismus und Konsistenz sowie Viskoelastizität und Abbaumechanismen [1]. Ursächlich für die Fremdkörperreaktion sind in den meisten Fällen die genuinen Materialeigenschaften der Fillersubstanz und/oder eine falsche Injektionstechnik (meist zu oberflächliche Applikation oder zu große Volumina an Fillersubstanz) [1]. Narins et al. unterteilt Nebenwirkungen von kosmetischen Fillern 
in Reaktionen vom Soforttyp, vom verzögerten Typ (2 Wochen bis 1 Jahr nach Injektion) und vom Spättyp, wobei Knötchen/Granulome in allen drei Kategorien auftreten können [2]. Viele der Komplikationen, die früher als Fremdkörpergranulome oder allergische Reaktionen angesehen wurden (bei negativer Bakterienkultur), sind nach Ansicht von Cassuto et al. auf sogenannte Biofilme zurückzuführen [3]. Als Biofilm wird ein Komplex aggregierter Mikroorganismen beschrieben, der sich durch Exkretion von extrazellulärer, schützender und adhäsiver Matrix kennzeichnet, resultierend in einer geringen Abwehrreaktion des Organismus, hoher antibiotischer Resistenz und schwieriger Nachweisbarkeit in Bakterienkulturen. Es wird vermutet, dass Biofilme durch Manipulation, Trauma oder Injektion anderer Substanzen in unmittelbarer Nähe aktiviert werden und zu lokaler (Abszess, Granuloma) bzw. systemischer Infektion führen können [3].

Fischer et al. publizierten 2007 einen Fall, in dem es bei einer Patientin 10 Jahre nach Injektion eines kosmetischen Permanent-Fillers (in beschriebenem Fall handelte es sich um Polymethylmethacrylat) unter antiviraler Therapie mit Peginterferon- $\alpha-2 a$ und Ribavirin, welche aufgrund einer chronischen Hepatitis $C$ eingeleitet wurde, zum Auftreten sarkoider Granulome im Bereich des injizierten Fillers kam [4]. Die Autoren beschrieben daher kosmetische permanente Filler als neue Kontraindikation für Interferon-Therapien [4].

In der Behandlung atropher Aknenarben wurde traditionell am häufigsten flüssiges Silikon verwendet. Dieses wird zu den permanenten Fillern gezählt und besteht aus Mikrosphären mit unregelmäßiger Oberfläche. Diese physikalische Eigenschaft verhindert die vollständige Phagozytose und führt vermehrt zu granulomatösen Fremdkörperreaktionen. Ebenso seit vielen Jahren im Einsatz ist der permanente Filler Hydroxyethylmethacrylat (HEMA)/Ethylmethacrylat (EMA) und Hyluronsäure (z. B. Dermalive $\left.^{\circledR}\right)$. HEMA und EMA bleiben jahrzehntelang in der Haut stabil und eine zu oberflächliche Injektion induziert häufig eine granulomatöse Fremdkörperreaktion [5]. Die histopathologischen Befunde aus läsionaler Haut mit derartigen Fillersubstanzen zeigen ein knotiges, granulomatöses Infiltrat in Dermis und Subkutis mit zahlreichen polygonalen, transluzierenden Fremdkörpern. Diese sind in der Polarisationsoptik typischerweise nicht doppelbrechend [5].

Welches Material genau im vorgestellten Falle verwendet wurde, wird jedoch spekulativ bleiben müssen.

Die therapeutischen Optionen sind bei einmal manifesten Granulomen enttäuschend [2]. Therapieziel ist dann v.a. eine Verminderung und Kontrolle der überschießenden lokalen Entzündungsreaktion. Bei inflammatorischen Granulomen ist eine topische und systemische Antibiose angezeigt, bei fluktuierenden Läsionen Inzision und Drainage der betroffenen Areale. Therapieversuche mit topischen Immunmodulatoren (Imiquimod, Pimecrolimus oder Tacrolimus) und intraläsionale Applikation von Steroiden oder 5-Fluorouracil sind möglich. Vielversprechend sind Therapieansätze mit Lasersystemen (Diodenlaser für granulomatöse Läsionen sowie Lithium-Triborat [LBO]-532-nm-Laser für inflammatorische Läsionen) [2]. Allopurinol kann in der Therapie sarkoider Granulome, des interstitiellen Granuloma anulare oder bei Tattoo-Reaktionen eingesetzt werden. Diskutiert wird ein möglicher Wirkmechanismus durch antioxidative bzw. antiinflammatorische Eigenschaften bzw. durch eine Verbesserung vaskulärer Funktionen [6,7]. Im vorgestellten Fall kam es jedoch zu keiner wegweisenden Befundbesserung unter oraler Allopurinolgabe. Bei frustraner Therapie und hohem subjektivem Leidensdruck ist als Ultima Ratio die chirurgische Exzision der Granulome erforderlich mit anschließender plastischer Rekonstrukion.

Der vorgestellte Fall erweitert das differenzialdiagnostische Spektrum unilateraler knotiger Hautläsionen im Gesicht und ist ein besonderes Beispiel für therapieinduzierte Spätfolgen (hier $>10$ Jahre). Wir halten das Wissen um die Langzeitnebenwirkungen der genannten kosmetischen Therapien für sehr wichtig, um künftig eine adäquate Patientenaufklärung zu gewährleisten, die hier ebenfalls nicht erfolgt war.

Interessenkonfilkt: Die Autoren geben an, dass kein Interessenkonflikt besteht.

\section{Abstract}

Granulomatous Foreign Body Granuloma as a Reaction to Previous Augmentation of Acne Scars - A Diagnostic and Therapeutic Hercules Challenge

$\nabla$

We report on a 45-years old asian woman who developed granulomatous reaction as a late onset side effect to cosmetic dermal filler injections. Due to the long time interval between injections and cutaneous reaction the diagnosis was only achieved through histologic examination. Treatment still poses a challenge as there are no standards established yet.

\section{Literatur}

1 Pavicic T. Fillers. An overview. Hautarzt. 2009; 60: 233-243 quiz 44

2 Narins RS, Coleman WP, Glogau RG. Recommendations and treatment options for nodules and other filler complications. Dermatol Surg 2009; 35 (Suppl 2): 1667-1671

3 Cassuto D, Marangoni O, De Santis G, Christensen L. Advanced laser techniques for filler-induced complications. Dermatol Surg 2009; 35 (Suppl 2): 1689-1695

4 Fischer J, Metzler G, Schaller M. Cosmetic permanent fillers for soft tissue augmentation: a new contraindication for interferon therapies. Arch Dermatol 2007; 143: 507-510

5 Requena L, Requena C, Christensen L et al. Adverse reactions to injectable soft tissue fillers. J Am Acad Dermatol 2011; 64: 1 - 34 quiz 5-6

6 Mazzatenta C, Ghilardi A, Grazzini M. Treatment of disseminated granuloma annulare with allopurinol: case report. Dermatol Ther 2010; 23 (Suppl 1): 24-27

7 Tsai TF, Yeh TY. Allopurinol in dermatology. Am J Clin Dermatol 2010; 11: $225-232$ 